\title{
Diagnostic yield of colon capsule endoscopy for Crohn's disease lesions in the whole gastrointestinal tract
}

\author{
Keisaku Yamada', Masanao Nakamura1*, Takeshi Yamamura' ${ }^{1}$ Keiko Maeda² ${ }^{1 *}$ Tsunaki Sawada², \\ Yasuyuki Mizutani ${ }^{1}$, Eri Ishikawa', Takuya Ishikawa', Naomi Kakushima', Kazuhiro Furukawa', Eizaburo Ohno', \\ Hiroki Kawashima², Takashi Honda', Masatoshi Ishigami' and Mitsuhiro Fujishiro'
}

\begin{abstract}
Background: Crohn's disease (CD) can involve the upper gastrointestinal (GI) tract as well as the small and large bowel. PillCam colon capsule endoscopy (PCCE-2) enables observation of the whole Gl tract, but its diagnostic yield for $\mathrm{CD}$ lesions in the whole $\mathrm{Gl}$ tract remains unknown.

Aim: To elucidate the diagnostic yield of PCCE-2 in patients with CD.

Methods: Patients with CD who underwent PCCE-2 and double-balloon endoscopy (DBE) using oral and anal approaches were evaluated for CD lesions in the whole $\mathrm{Gl}$ tract. We divided the small bowel into three segments (jejunum, ileum, and terminal ileum), and the large bowel into four segments (right colon, transverse colon, left colon, rectum). Detection of ulcer scars, erosion, ulcers, bamboo joint-like appearance, and notch-like appearance was assessed in each segment. The diagnostic yield of PCCE-2 was analyzed based on the DBE results as the gold standard.

Results: Of the total 124 segments, the sensitivities of PCCE-2 for ulcer scars, erosion, and ulcers were 83.3\%, 93.8\%, and $88.5 \%$, respectively, and the specificities were $76.0 \%, 78.3 \%$, and $81.6 \%$, respectively. For the 60 small bowel segments, the sensitivities were $84.2 \%, 95.5 \%$, and $90.0 \%$, respectively, and the specificities were $63.4 \%, 86.8 \%$, and $87.5 \%$, respectively. For the 64 large bowel segments, the sensitivities were $80.0 \%, 90.0 \%$, and $83.3 \%$, respectively, and the specificities were $84.7 \%, 72.2 \%$, and $77.6 \%$, respectively.
\end{abstract}

Conclusion: PCCE-2 provides a high diagnostic yield for lesions in the whole Gl tract of patients with CD. Thus, we recommend its use as a pan-enteric tool in clinical settings.

Keywords: Colon capsule endoscopy, Crohn's disease, Diagnostic yield, Retention, Whole gastrointestinal tract, Prospective study

\section{Introduction}

Crohn's disease $(C D)$ is a chronic inflammatory bowel disease (IBD) that mainly involves the small and large bowel. The goals of treatment in $\mathrm{CD}$ have evolved

\footnotetext{
*Correspondence: makamura@med.nagoya-u.ac.jp

1 Department of Gastroenterology and Hepatology, Nagoya University Graduate School of Medicine, 65 Tsurumai-cho, Syowa-ku, Nagoya City, Aichi, Japan

Full list of author information is available at the end of the article
}

in recent years from symptom control to healing of mucosal lesions visualized on endoscopy [1]. Mucosal healing has been associated with improved clinical outcomes, including sustained steroid-free clinical remission, decreased rates of surgery and hospitalization, reduced occurrence of new perianal complications, as well as improvement in quality of life and increased work productivity [2,3]. With the development of small bowel capsule endoscopy (SBCE), direct 
endoscopic examination of the whole small bowel mucosa is available with high diagnostic yield [4-6]. However, in addition to SBCE, colonoscopy (CS) is required to evaluate the large-bowel mucosa, particularly in the case of ileocolonic CD. The introduction of a minimally invasive and high-performing tool for evaluating the whole gastrointestinal (GI) tract is highly anticipated, because even with balloon-assisted enteroscopy, it can be challenging to examine the whole GI tract.

The second-generation PillCam colon capsule endoscope (PCCE-2; Medtronic Co. Ltd., Dublin, Ireland) has been developed and can be used to examine the whole GI tract, including the small and large bowel, although it was originally designed as a tool to diagnose colorectal lesions [6]. PCCE-2 is a noninvasive procedure that enables visualization of the GI tract without sedation or gas insufflation [7]. The feasibility and safety of its use in colonic assessment have been investigated for polyps and cancer [8-11]. Furthermore, PCCE-2 has been clinically applied as a tool to replace CS for observing the large bowel in patients with ulcerative colitis (UC) [10], and an original preparation regimen has been developed [11, 12]. Although some studies confirmed the safety and feasibility of PCCE-2 as a pan-enteric tool for patients with $\mathrm{CD}[6,13]$, the diagnostic yield of PCCE-2 for the whole GI CD lesions remains unknown. On the other hand, balloon-assisted enteroscopy, such as double-balloon endoscopy (DBE) and single-balloon endoscopy (SBE), has been shown to have a high diagnostic yield in detecting small bowel diseases [14]. SBE results have been reported as the gold standard for small bowel lesions of CD [15]. Therefore, the aim of this study was to prospectively elucidate the diagnostic yield of PCCE-2 for the whole GI $\mathrm{CD}$ lesions in reference to $\mathrm{DBE}$ results.

\section{Materials and methods}

Patients

From June 2018 to August 2019, patients who were scheduled for DBE for assessment of CD activity at the Nagoya University Hospital were enrolled in this prospective study. The study protocol was approved by the local ethics committee (Nagoya University IRB 2015372) and registered at UMIN-CTR (UMIN000019632). Patients provided informed consent. This study was conducted in accordance with the principles of the Declaration of Helsinki.

\section{Study protocol}

The details of the study protocol are shown in Table 1 . On the first day, transoral DBE was performed, and patients who showed no stricture on DBE proceeded to PCCE-2 preparation. In addition to PCCE-2 preparation, patency of the GI tract was assessed using the PillCam patency capsule (PC) (Medtronic Co. Ltd., Dublin, Ireland). PCCE-2 was not performed for patients in whom patency was not confirmed. After the excretion of PCCE2 , trans-anal DBE was performed. The primary endpoint of this study was the diagnostic yield of PCCE-2 for erosive lesions in each segment, and the secondary endpoints were the evaluation of CD activity using PCCE-2 and clinical results of PCCE-2 including retention of capsule.

\section{Definitions of DBE and PCCE-2 recordings}

The whole GI tract was observed on both DBE and PCCE-2 recordings. Whole GI tract observation with DBE was defined as when the landmark CD lesion was observed using both oral and anal approaches, or several endoscopists judged that DBE enabled visualization of the whole GI tract using fluoroscopy and additional gastrografin enterography during $\mathrm{DBE}$, according to

Table 1 Original regimen of PCCE-2 plus DBE examinations

\begin{tabular}{|c|c|c|}
\hline Day & Time & Procedure \\
\hline \multirow[t]{2}{*}{ Day 1} & Daytime & DBE oral approach \\
\hline & Before bedtime & 2 senna tablets and $P C$ \\
\hline Day 2 & Before bedtime & $\begin{array}{l}\text { Magnesium citrate } 50 \mathrm{~g}(180 \mathrm{ml}) \text { : hypertonic method } \\
2 \text { senna tablets }\end{array}$ \\
\hline \multirow[t]{6}{*}{ Day 3} & $8: 30$ & 1.0 L PEG and patency confirmation \\
\hline & $10: 00$ & Capsule ingestion with mosapride citrate 20 mg \\
\hline & 1st boost (when the PCCE-2 reaches the small intestine) & $\begin{array}{l}\text { A mixture of PEG } 1000 \mathrm{ml} \text { and water } 1000 \mathrm{ml} \\
\text { Castor oil } 30 \mathrm{ml}\end{array}$ \\
\hline & 2nd boost & Sodium picosulfate 48 mg Castor oil $30 \mathrm{ml}$ \\
\hline & 3rd boost & Magnesium citrate $50 \mathrm{~g}(900 \mathrm{ml})$ : isotonic method \\
\hline & (after the excretion of PCCE-2) & DBE anal approach \\
\hline
\end{tabular}

PCCE-2 the second-generation PillCam colon capsule endoscopy, DBE double-balloon endoscopy, $P C$ patency capsule, $P E G$ polyethylene glycol 
previous studies $[16,17]$ (Fig. 1). In cases where the CCE was not excreted, $C D$ lesions were analyzed as far as the CCE reached. Two readers who were blinded to the clinical background and DBE findings of the patient analyzed each PCCE-2 video.

We divided the small bowel into three segments, namely, the jejunum, ileum, and terminal ileum, and the large bowel into four segments, namely, the right side of the colon (cecum, ascending colon), transverse colon, and left side of the colon (descending colon, sigmoid colon), and rectum (Fig. 2). The terminal ileum was defined as the Sect. $10 \mathrm{~cm}$ from the ileocecal valve on DBE, and the video segment $5 \mathrm{~min}$ before the cecum was reached on PCCE-2. In the small bowel postoperative cases, the remaining bowel was divided into three segments as

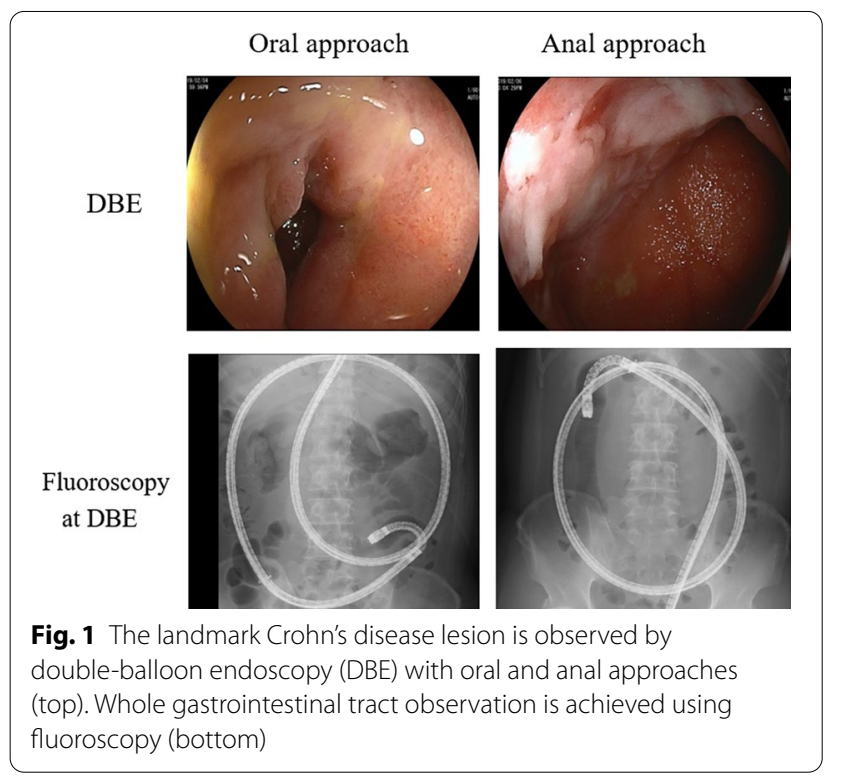

previously defined. The diagnostic yield of PCCE-2 for the presence of ulcer scars, erosion, and ulcers in each segment was evaluated in the seven segments with the DBE results defined as the reference gold standard. We focused on evaluating the presence of any lesion in each segment. In cases with several lesions in one segment, we confirmed that the segment was positive. The number of positive segments in DBE and PCCE-2 were compared. PCCE-2 findings were also evaluated for esophageal and gastric lesions using the same strategy. According to $\mathrm{CD}$ activity, SES-CD was evaluated right after DBE. SES-CD originally involves the terminal ileum and rectum, and in this study, it was applied for the jejunum and ileum using the same evaluation method. Total score, the sum of SESCD in each segment, was described as modified SES-CD. Capsule endoscopy Crohn's disease activity index (CECDAI), frequently used to evaluate $C D$ activity in small bowel capsule endoscopy, was used to evaluate the seven segments. The total score, the sum of CECDAI in each segment of PCCE-2, was described as modified CECDAI in this study. The relation between modified SES-CD and modified CECDAI was analyzed in the same patients.

\section{Colon cleansing level}

Colon cleanliness was determined in accordance with a four-point grading scale (excellent, good (categorized as adequate), fair, and poor (categorized as inadequate)) as reported in a previous study [18].

\section{Statistical analysis}

All data were analyzed using SPSS version 24.0 statistical software (IBM, Tokyo, Japan). Differences in each segment of the small and large bowel were analyzed using Fisher's exact test. The factors that influenced the incomplete PCCE-2 were analyzed using a logistic regression model. To analyze the relation between modified SES-CD

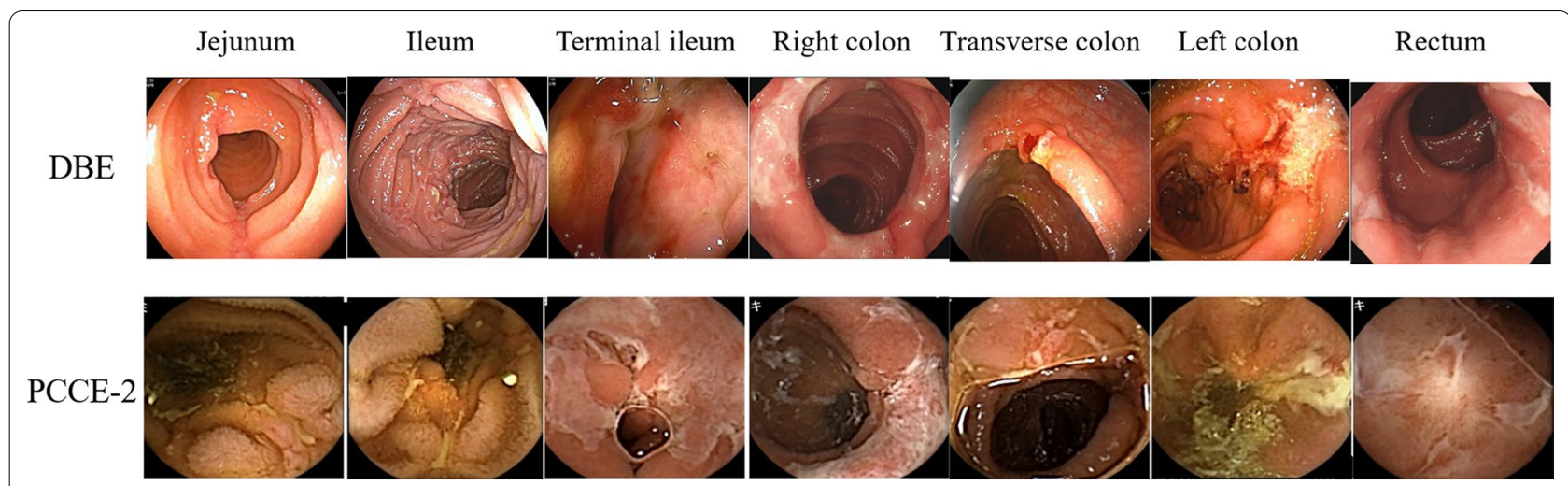

Fig. 2 Images of double-balloon endoscopy (top) and the corresponding second-generation PillCam colon capsule endoscopy (PCCE-2) image (bottom) of the ulcer lesion 
and modified CECDAI, Spearman's rank correlation coefficient was used. Differences with a $p$-value $<0.05$ were considered statistically significant.

\section{Results}

\section{Patients}

A total of 22 patients were enrolled and underwent DBE using an oral approach. Small bowel obstruction was suspected in one patient, and patency was not confirmed by PC in another patient. Finally, 20 patients underwent PCCE-2 and subsequent DBE using an anal approach. Table 2 shows the characteristics of the 20 patients.

\section{Results of the PCCE-2 procedure and number of segments} evaluated by both modalities

The PCCE-2 excretion rate within the battery life was $75 \%(15 / 20)$. Of the five patients who did not excrete the PCCE-2, two were observed up to the left colon, one was observed up to the transverse colon, and two were observed up to the right colon. Of the 15 patients who excreted the PCCE-2, the median duration of the entire examination was $455 \mathrm{~min}$, the gastric transit time was $80 \mathrm{~min}$, the small intestinal transit time was $69 \mathrm{~min}$, and the colorectal transit time was $265 \mathrm{~min}$. The colon cleansing level was evaluated as adequate in $80 \%$ of patients.

Of the 20 patients, 20 gastric, 60 small bowel, and 64 large bowel segments were evaluated. As for large bowel segments, 16 segments were excluded because PCCE-2 could not be observed or evaluated in postoperative cases.

\section{Positive findings of PCCE-2 in the whole GI tract}

Various lesions, including ulcer scars, erosion, ulcers, bamboo joint-like appearance, and notch-like appearance, were detected in the whole GI tract of patients

Table 2 Patient characteristics $(\mathbf{N}=\mathbf{2 0})$

\begin{tabular}{lll}
\hline Age (years) & Median, range & $\mathbf{3 5}(\mathbf{1 9 - 6 7 )}$ \\
\hline Sex & Male/female & $15 / 5$ \\
Disease duration (years) & Median, range & $10(1-22)$ \\
Disease location & L1/L2/L3 $3^{\mathrm{a}}$ & $6 / 0 / 14$ \\
CDAl & Median, range & $142(60-324)^{\mathrm{b}}$ \\
History of surgery (\%) & & $70.0 \%(14 / 20)$ \\
Medication (\%) & Mesalazine & $80.0 \%(16 / 20)$ \\
& Elemental diet & $70.0 \%(14 / 20)$ \\
& Anti-TNF agents & $55.0 \%(11 / 20)$ \\
& Thiopurines & $25.0 \%(5 / 20)$ \\
& Ustekinumab & $10.0 \%(2 / 20)$ \\
\hline
\end{tabular}

CDAl Crohn's disease activity index, TNF tumor necrosis factor

a L1/L2/L3: ileal/colonic/ileocolonic, Montreal classification

${ }^{b}$ CDAl could not be evaluated in one patient because of the presence of stoma with CD by PCCE-2. Among them, ulcer scars, erosion, and ulcers were frequently observed in the small and large bowel, and erosion and bamboo joint-like appearance were most commonly observed in the stomach. The detection rates of PCCE-2 for ulcer scars, erosion, and ulcers per segment were $52 \%, 43.3 \%$, and $10 \%$ in the small bowel, and $20 \%, 38 \%$, and $28 \%$, respectively, in the large bowel. The detection rates for erosion and bamboo joint-like appearance in the stomach were $35 \%$ and $10 \%$, respectively.

\section{Diagnostic yield of PCCE-2}

The diagnostic yield of PCCE-2 for the small and large bowel is shown in Table 3. The PCCE-2 sensitivities for ulcer scars, erosion, and ulcers were $83.3 \%, 93.8 \%$, and $88.5 \%$, respectively, and the specificities were $76.0 \%$, $78.3 \%$, and $81.6 \%$, respectively.

The diagnostic yield of PCCE-2 for the small bowel is shown in Table 4. Sensitivities and specificities for active $\mathrm{CD}$ lesions, such as erosion and ulcers, were more than $85 \%$. No significant difference was found in the sensitivities and specificities between the three segments of the small bowel (Table 5).

Table 6 shows the diagnostic yield of PCCE-2 for the large bowel. The sensitivities were satisfactory, but the specificities for erosion and ulcers were relatively low compared with those of the small bowel. No significant difference was observed between the four segments of the large bowel, as was found for the small bowel

Table 3 Diagnostic yield of PCCE-2 for the small and large bowel

\begin{tabular}{llll}
\hline & Ulcer scar & Erosion & Ulcer \\
\hline Sensitivity & $83.3 \%(20 / 24)$ & $93.8 \%(30 / 32)$ & $88.5 \%(23 / 26)$ \\
Specificity & $76.0 \%(76 / 100)$ & $78.3 \%(72 / 92)$ & $81.6 \%(80 / 98)$ \\
PPV & $45.5 \%(20 / 44)$ & $60.0 \%(30 / 50)$ & $56.1 \%(23 / 41)$ \\
NPV & $95.0 \%(76 / 80)$ & $97.3 \%(72 / 74)$ & $96.4 \%(80 / 83)$ \\
Accuracy & $77.4 \%(96 / 124)$ & $82.3 \%(102 / 124)$ & $83.1 \%(103 / 124)$ \\
\hline
\end{tabular}

$P P V$ positive predictive value, NPV negative predictive value, $P C C E-2$ the secondgeneration PillCam colon capsule endoscopy

Table 4 Diagnostic yield of PCCE-2 for the small bowel

\begin{tabular}{llll}
\hline & Ulcer scar & Erosion & Ulcer \\
\hline Sensitivity & $84.2 \%(16 / 19)$ & $95.5 \%(21 / 22)$ & $90.0 \%(18 / 20)$ \\
Specificity & $63.4 \%(26 / 41)$ & $86.8 \%(33 / 38)$ & $87.5 \%(35 / 40)$ \\
PPV & $51.6 \%(16 / 31)$ & $80.8 \%(21 / 26)$ & $78.3 \%(18 / 23)$ \\
NPV & $89.7 \%(26 / 29)$ & $97.1 \%(33 / 34)$ & $94.6 \%(35 / 37)$ \\
Accuracy & $70.0 \%(42 / 60)$ & $90.0 \%(54 / 60)$ & $88.3 \%(53 / 60)$ \\
\hline
\end{tabular}

$P P V$ positive predictive value, NPV negative predictive value, $P C C E-2$ the secondgeneration PillCam colon capsule endoscopy 
Table 5 Diagnostic yield of PCCE-2 for the small bowel by segment

\begin{tabular}{|c|c|c|c|c|}
\hline & Jejunum & Ileum & Terminal ileum & $p$ value \\
\hline \multicolumn{5}{|l|}{ Ulcer scar } \\
\hline Sensitivity & $100 \%(5 / 5)$ & $75.0 \%(6 / 8)$ & $83.3 \%(5 / 6)$ & n.s.* \\
\hline Specificity & $53.3 \%(8 / 15)$ & $66.7 \%(8 / 12)$ & $71.4 \%(10 / 14)$ & n.s.* \\
\hline \multicolumn{5}{|l|}{ Erosion } \\
\hline Sensitivity & $100 \%(4 / 4)$ & $90.0 \%(9 / 10)$ & $100 \%(8 / 8)$ & n.s.* \\
\hline Specificity & $93.8 \%(15 / 16)$ & $90.0 \%(9 / 10)$ & $75.0 \%(9 / 12)$ & n.s.* \\
\hline \multicolumn{5}{|l|}{ Ulcer } \\
\hline Sensitivity & $100 \%(4 / 4)$ & $87.5 \%(7 / 8)$ & $87.5 \%(7 / 8)$ & n.s.* \\
\hline Specificity & $87.5 \%(14 / 16)$ & $83.3 \%(10 / 12)$ & $83.3 \%(10 / 12)$ & n.s.* \\
\hline
\end{tabular}

PCCE-2 the second-generation PillCam colon capsule endoscopy *Fisher's exact test

Table 6 Diagnostic yield of PCCE-2 for the large bowel

\begin{tabular}{llll}
\hline & Ulcer scar & Erosion & Ulcer \\
\hline Sensitivity & $80.0 \%(4 / 5)$ & $90.0 \%(9 / 10)$ & $83.3 \%(5 / 6)$ \\
Specificity & $84.7 \%(50 / 59)$ & $72.2 \%(39 / 54)$ & $77.6 \%(45 / 58)$ \\
PPV & $30.8 \%(4 / 13)$ & $37.5 \%(9 / 24)$ & $27.8 \%(5 / 18)$ \\
NPV & $98.0 \%(50 / 51)$ & $97.5 \%(39 / 40)$ & $97.8 \%(45 / 46)$ \\
Accuracy & $84.4 \%(54 / 64)$ & $75.0 \%(48 / 64)$ & $78.1 \%(50 / 64)$ \\
\hline
\end{tabular}

PPV positive predictive value, NPV negative predictive value, $P C C E-2$ the secondgeneration PillCam colon capsule endoscopy

(Table 7). Modified CECDAI was well related to modified SES-CD (Fig. 3) (Spearman's rank correlation coefficient, $\mathrm{rho}=0.935, p<0.001)$.

For gastric lesions, erosion and bamboo joint-like appearance were detected. Additional file 1: Table S1 shows the diagnostic yield of these gastric lesions. No esophageal lesions were noted in any of the patients.
Incidence and severity of PCCE-2 procedure-related adverse events

PCCE-2 retention was not observed in patients with $\mathrm{CD}$ in whom patency was confirmed by PC. Of the 20 patients, one patient had moderate and three had mild abdominal bloating, two had mild abdominal pain, and two had mild nausea during PCCE-2 examination. Eighteen of the 20 patients indicated that they would undergo PCCE-2 again, and they preferred PCCE-2 to CS and DBE.

\section{Discussion}

PCCE-2 can observe CD lesions that involve the whole GI tract in a single examination. Although some studies have reported the safety and feasibility of PCCE- 2 for patients with CD $[13,15]$, the diagnostic yield of PCCE-2 for CD lesions of the whole GI is still unknown. This study is the first to elucidate the diagnostic yield of PCCE-2 for the whole GI tract. We demonstrated that PCCE-2 has high diagnostic yield for $\mathrm{CD}$ lesions of the whole GI.

With regard to the small bowel, a number of studies have already reported on the diagnostic yield of SBCE for small bowel CD lesions. The results of these studies vary with sensitivities of approximately $80 \%$ and specificities of approximately $50-75 \%[19,20]$. Solem et al. [19] reported that the specificity for small bowel CD lesions is significantly lower with SBCE than with other small bowel imaging modalities, such as CT enterography, ileocolonoscopy, and small bowel follow-through. We showed that the sensitivity and specificity of PCCE-2 for small bowel ulcer lesions were $90.0 \%$ and $87.5 \%$, respectively. We can conclude that the specificity of PCCE-2 is high, suggesting that PCCE-2 may reduce false positives. The higher diagnostic accuracy of PCCE-2 has several potential explanations. First, the PCCE-2 has two head cameras, each with a $172^{\circ}$ angle of view, allowing for almost $360^{\circ}$ visual coverage of the colon. Second, PCCE-2

Table 7 Diagnostic yield of PCCE-2 for the large bowel by segment

\begin{tabular}{|c|c|c|c|c|c|}
\hline & Right colon & Transverse colon & Left colon & Rectum & $p$ value \\
\hline \multicolumn{6}{|l|}{ Ulcer scar } \\
\hline Sensitivity & $100 \%(1 / 1)$ & $50 \%(1 / 2)$ & $100 \%(1 / 1)$ & $100 \%(1 / 1)$ & n.s. ${ }^{*}$ \\
\hline Specificity & $86.7 \%(13 / 15)$ & $86.7 \%(13 / 15)$ & $86.7 \%(13 / 15)$ & $84.6 \%(11 / 13)$ & n.s.* \\
\hline \multicolumn{6}{|l|}{ Erosion } \\
\hline Sensitivity & $100 \%(3 / 3)$ & $100 \%(3 / 3)$ & $66.7 \%(2 / 3)$ & $100 \%(1 / 1)$ & n.s. ${ }^{*}$ \\
\hline Specificity & $69.2 \%(9 / 13)$ & $64.3 \%(9 / 14)$ & $78.6 \%(11 / 14)$ & $76.9 \%(10 / 13)$ & n.s.* \\
\hline \multicolumn{6}{|l|}{ Ulcer } \\
\hline Sensitivity & $100 \%(1 / 1)$ & $66.7 \%(2 / 3)$ & $100 \%(1 / 1)$ & $100 \%(1 / 1)$ & n.s. ${ }^{*}$ \\
\hline Specificity & $80.0 \%(12 / 15)$ & $78.6 \%(11 / 14)$ & $75.0 \%(12 / 16)$ & $76.9 \%(10 / 13)$ & n.s.* \\
\hline
\end{tabular}

PCCE-2 the second-generation PillCam colon capsule endoscopy

*Fisher's exact test 


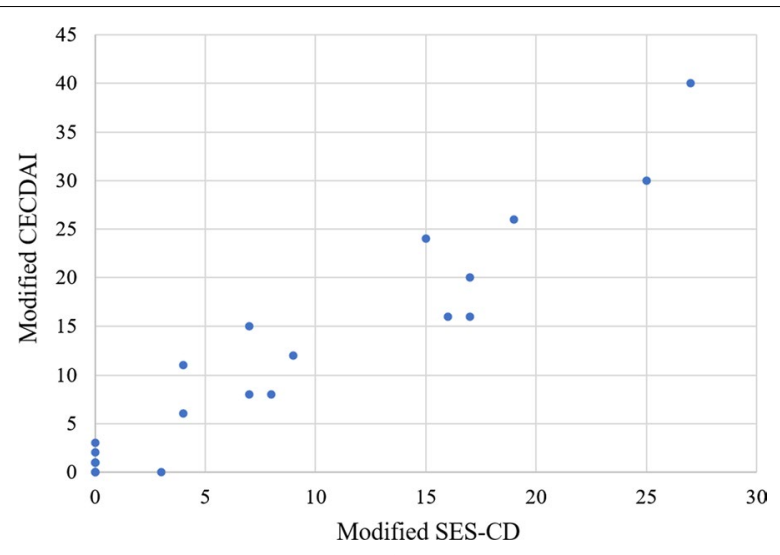

Fig. 3 The relation between modified SES-CD and modified CECDAI. Modified CECDAI was well related to modified SES-CD (Spearman's rank correlation coefficient, rho $=0.935, p<0.001$ )

has improved image acquisition and adaptive frame rates of 4 to 35 images per second [21,22], which are much higher than the 2 to 6 image frame rates of SBCE. Therefore, the performance of PCCE-2 could improve the diagnostic yield for small bowel lesions. Furthermore, the use of laxatives has been reported to be beneficial in patients likely to have subtle findings on SBCE because laxatives improve small bowel visualization quality [23]. In this study, the regular bowel preparation before PCCE-2 ingestion may also have contributed to the improved diagnostic yield.

The specificities of PCCE-2 for erosion and ulcers of the large bowel were $72.2 \%$ and $77.6 \%$, respectively, which were lower than those of the small bowel. D'Haens et al. [1] stated that the low specificity may be related to bowel preparation, with adherent stools being erroneously identified as ulcerations (Fig. 4). With regard to the diagnostic yield of the large bowel, there is still room for improvement.

In addition to the observation of the small and large bowel, PCCE-2 can also observe upper GI lesions simultaneously. In patients with $\mathrm{CD}$, gastric lesions, such as erosion, ulcers, and bamboo joint-like appearance, are detected at a relatively high frequency (24-73\%) [24], and bamboo joint-like appearance is a characteristic finding in the upper GI. Fujiya et al. [25] reported that the detection rates of this finding are $38.3 \%$ in patients with $\mathrm{CD}$, $2.5 \%$ in gender- and age-matched patients without IBD, and $1.5 \%$ in patients with UC. Therefore, PCCE- 2 may be helpful in diagnosing patients with $C D$ by distinguishing them from patients without IBD and patients with UC.

Furthermore, we demonstrated that the PCCE-2 procedure is safe for patients with CD. CD is a chronic inflammatory disease, and patients with $\mathrm{CD}$ need to undergo repeated GI examinations. Therefore, examination tools that are highly acceptable with fewer complications are desired. PCCE-2 retention was not observed in patients with $\mathrm{CD}$ in whom patency was confirmed by $\mathrm{PC}$, and a high percentage of patients $(18 / 20)$ indicated that they would undergo PCCE-2 again and preferred PCCE-2 to CS and DBE.

The use of castor oil (Himashi Oil; Yoshida Pharmaceutical, Tokyo, Japan) as part of the PCCE-2 regimen has been widely established as the standard regimen for bowel preparation because it improves capsule excretion rate and shortens capsule transit time [26, 27]. Although castor oil was also used in this study, the excretion rate of PCCE-2 was not sufficient. This was attributed to the
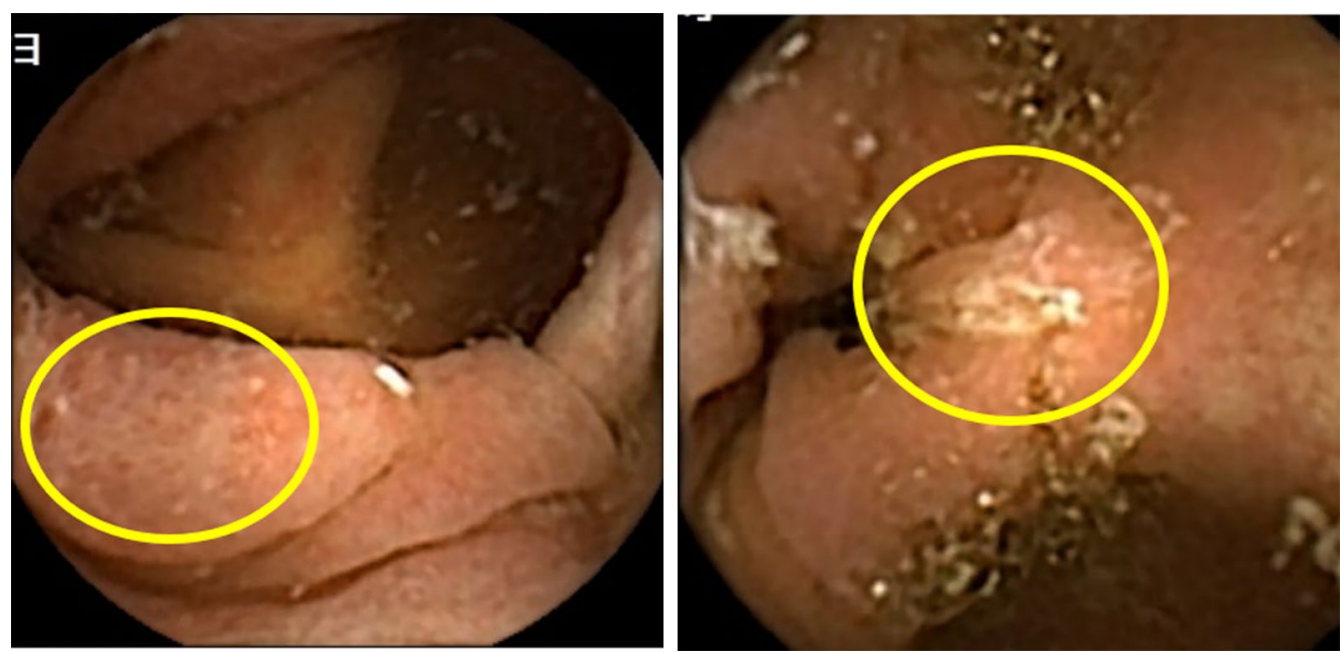

Fig. 4 False-positive cases: stools may be erroneously identified as erosion and ulcer 
limited PCCE-2 examination time because DBE using an anal approach had to be performed later. Another reason was inflammation of the GI tract due to CD. In patients with UC, the presence of colonic mucosal inflammation has been reported to correlate with longer PCCE-2 transit times because inflammation decreases the motility of the PCCE-2 [12]. Additional file 1: Table S2 shows the modified SES-CD score for small and large bowel inflammation (details are described in Additional file 1: Table S2), which was identified as a factor resulting in incomplete PCCE-2. Therefore, PCCE-2 seemed to be less likely to be excreted in patients with $C D$ with an active lesion compared with patients with UC. Furthermore, postoperative cases tended to be classified more often to the non-excretion group, despite the short bowel. A possible reason was that the PCCE-2 moved around in a few cases for several hours at the anastomosis and did not flow to the anal side because of local intestinal peristalsis.

However, in contrast to UC that affects the colon in a retrograde and continuous manner starting from the rectum and extending proximally [28], CD involves discontinuous lesions, and the most common site is the terminal ileum [29]. Although five patients with CD did not excrete the PCCE-2 in this study, the terminal ileum could be observed in all cases, and no CD lesions were observed on DBE at the segment that could not be observed by PCCE-2. Therefore, even if the PCCE- 2 is not excreted, the mucosal evaluation of $\mathrm{CD}$ is considered sufficient as PCCE-2 contributes to the evaluation of CD activity in cases with available PCCE- 2 .

\section{Limitations}

The main limitation of this study was the small number of patients enrolled and the low number of patients with large bowel CD lesions. However, no studies have compared PCCE-2 results with DBE findings for the whole GI tract in patients with CD. Therefore, this study contributes significantly to the evidence supporting the clinical usefulness of the PCCE-2 as a pan-enteric tool for evaluating CD. PCCE-2 was usually performed 2 days after oral DBE. Then, we evaluated the jejunal lesions in PCCE-2 carefully, with due consideration of traumatic mucosal injury. However, suspected traumatic lesions such as reddish area and linear mucosal damage were few in the jejunum and could be differentiated with $C D$ lesions. Whole GI tract observation was evaluated using the landmark CD lesion, fluoroscopy, and gastrografin enterography; however, the marking clip placement was considered to be more accurate for evaluation.

In conclusion, PCCE-2 is a safe and feasible tool to examine the whole GI tract in patients with CD with a high diagnostic yield for CD lesions in the entire GI.
Further large-scale studies are required for an in-depth understanding of the usefulness of PCCE-2 for CD.

\section{Supplementary Information}

The online version contains supplementary material available at https://doi. org/10.1186/s12876-021-01657-0.

Additional file 1. Supplementary Table 1: Diagnostic yield of PCCE-2 for gastric lesion. Supplementary Table 2: Univariate logistic analysis of factors influencing incomplete PCCE-2.

\section{Acknowledgements \\ None.}

Authors' contributions

Conception and design: YK, NM; analysis and interpretation of the data: YK, NM, YT, MK, ST, MY, IE, FK; capsule endoscopy reading: YK, NM; drafting of the article: NM, YK; statistical analysis: NM; critical revision of the article for important intellectual content: IT, OE, HT, KH, IM, KN; final approval of the article: FM. All authors read and approved the final manuscript.

\section{Funding}

None.

\section{Availability of data and materials}

The datasets used and/or analyzed during the current study are available from the corresponding author on reasonable request.

\section{Ethics approval and consent to participate}

The study protocol was approved by the local ethics committee (Nagoya University Hospital ethic committee ID 2015-372), registered at UMIN-CTR (UMIN000019632), and written informed consent was obtained from all patients who met the inclusion criteria and agreed to participate in the study. The work performed in this study was in accordance with the principles of the Declaration of Helsinki.

\section{Consent for publication}

Not applicable.

\section{Competing interests}

The authors declare that they have no conflict of interest.

\section{Author details}

${ }^{1}$ Department of Gastroenterology and Hepatology, Nagoya University Graduate School of Medicine, 65 Tsurumai-cho, Syowa-ku, Nagoya City, Aichi, Japan. ${ }^{2}$ Department of Endoscopy, Nagoya University Hospital, 65 Tsurumai-cho, Showa-ku, Nagoya 466-8550, Japan.

Received: 1 December 2020 Accepted: 18 January 2021

Published online: 16 February 2021

\section{References}

1. D'Haens G, Löwenberg M, Samaan MA, Franchimont D, Ponsioen C, van den Brink GR, et al. Safety and feasibility of using the second-generation Pillcam colon capsule to assess active colonic Crohn's disease. Clin Gastroenterol Hepatol. 2015;13:1480-6.e3.

2. Frøslie KF, Jahnsen J, Moum BA, Vatn M, IBSEN Group. Mucosal healing in inflammatory bowel disease: results from a Norwegian population-based cohort. Gastroenterology 2007;133:412-22.

3. Casellas F, Barreiro De Acosta M, Iglesias M, Robles V, Nos P, et al. Mucosal healing restores normal health and quality of life in patients with inflammatory bowel disease. Eur J Gastroenterol Hepatol. 2012;24:762-9.

4. Kopylov U, Seidman EG. Clinical applications of small bowel capsule endoscopy. Clin Exp Gastroenterol. 2013;6:129-37. 
5. Park SK, Ye BD, Kim KO, Park CH, Lee WS, Jang Bl, et al. Guidelines for video capsule endoscopy: emphasis on Crohn's disease. Clin Endosc. 2015;48:128-35

6. Carvalho PB, Rosa B, De Castro FD, Moreira MJ, Cotter J. PillCam COLON $2 \Subset$ in Crohn's disease: a new concept of pan-enteric mucosal healing assessment. World J Gastroenterol. 2015;21:7233-41.

7. Schoofs N, Devière J, Van Gossum A. PillCam colon capsule endoscopy compared with colonoscopy for colorectal tumor diagnosis: a prospective pilot study. Endoscopy. 2006;38:971-7.

8. Van Gossum A, Munoz-Navas M, Fernandez-Urien I, Carretero C, Gay G, Delvaux M, et al. Capsule endoscopy for the detection of polyps and cancer. N Engl J Med. 2009;361:264-70.

9. Yamada K, Nakamura M, Yamamura T, Maeda K, Sawada T, Mizutani Y, et al. Clinical factors associated with missing colorectal polyp on colon capsule endoscopy. Digestion. 2020;101:316-22.

10. Hosoe N, Nakano M, Takeuchi K, Endo Y, Matsuoka K, Abe T, et al. Establishment of a novel scoring system for colon capsule endoscopy to assess the severity of ulcerative colitis-Capsule scoring of ulcerative colitis. Inflamm Bowel Dis. 2018;24:2641-7.

11. Shi HY, Chan FKL, Higashimori A, Kyaw M, Ching JYL, Chan $\mathrm{HCH}$, et al. A prospective study on second-generation colon capsule endoscopy to detect mucosal lesions and disease activity in ulcerative colitis (with video). Gastrointest Endosc. 2017;86:1139-46.e6.

12. Okabayashi S, Kobayashi T, Nakano M, Toyonaga T, Ozaki R, Tablante MC, et al. A simple 1-day colon capsule endoscopy procedure demonstrated to be a highly acceptable monitoring tool for ulcerative colitis. Inflamm Bowel Dis. 2018:24:2404-12

13. Hall B, Holleran G, MCNamara D. PillCam COLON 2 @ as a pan-enteroscopic test in Crohn's disease. World J Gastrointest Endosc. 2015;7:1230-2.

14. Pasha SF, Leighton JA, Das A, Harrison ME, Decker GA, Fleischer DE, et al. Double-balloon enteroscopy and capsule endoscopy have comparable diagnostic yield in small-bowel disease: a meta-analysis. Clin Gastroenterol Hepatol. 2008:6:671-6.

15. Takenaka K, Ohtsuka K, Kitazume Y, Nagahori M, Fujii T, Saito E, et al. Comparison of magnetic resonance and balloon enteroscopic examination of the small intestine in patients with Crohn's disease. Gastroenterology. 2014;147:334-42.e3

16. Hattori S, Nakamura M, Yamamura T, Maeda K, Sawada T, Mizutani Y, et al. The microbiome can predict mucosal healing in small intestine in patients with Crohn's disease. J Gastroenterol. 2020;55:1138-49.

17. Matsuura R, Watanabe $O$, Nakamura M, Yamamura T, Matsushita M, Suhara $\mathrm{H}$, et al. Fecal calprotectin reflects endoscopic activity in patients with small-bowel Crohn's disease according to double-balloon endoscopy findings. Nagoya J Med Sci. 2018;80:257-66.
18. Zhou J, Tang X, Wang J, Chen Z, Wang X, Jiang B. Feasibility of a novel low-volume and sodium phosphate-free bowel preparation regimen for colon capsule endoscopy. Exp Ther Med. 2017;14:1739-43.

19. Solem CA, Loftus EV, Fletcher JG, Baron TH, Gostout CJ, Petersen BT, et al. Small bowel imaging in Crohn's disease: a prospective, blinded, 4-way comparison trial. Gastrointest Endosc. 2008;68:255-66.

20. Aloi M, Di NG, Romano G, Casciani E, Civitelli F, Oliva S, et al. Magnetic resonance enterography, small-intestine contrast US, and capsule endoscopy to evaluate the small bowel in pediatric Crohn's disease: a prospective, blinded, comparison study. Gastrointest Endosc. 2015;81:420-7.

21. Tal AO, Vermehren J, Albert JG. Colon capsule endoscopy: current status and future directions. World J Gastroenterol. 2014;20:16596-602.

22. Eliakim R, Yassin K, Niv Y, Metzger Y, Lachter J, Gal E, Sapoznikov B, et al. Prospective multicenter performance evaluation of the secondgeneration colon capsule compared with colonoscopy. Endoscopy. 2009:41:1026-31.

23. Yung DE, Rondonotti E, Sykes C, Pennazio M, Plevris JN, Koulaouzidis A. Systematic review and meta-analysis: is bowel preparation still necessary in small bowel capsule endoscopy? Expert Rev Gastroenterol Hepatol. 2017;11:979-93.

24. Nomura Y, Moriichi K, Fujiya M, Okumura T. The endoscopic findings of the upper gastrointestinal tract in patients with Crohn's disease. Clin J Gastroenterol. 2017;10:289-96.

25. Fujiya M, Sakatani A, Dokoshi T, Tanaka K, Ando K, Ueno N, et al. A bamboo joint-like appearance is a characteristic finding in the upper gastrointestinal tract of Crohn's disease patients: a case-control study. Medicine. 2015;94:e1500.

26. Hotta N. The use of castor oil for bowel preparation for colon capsule endoscopy. Open J Med Imaging. 2016;6:103-7.

27. Ohmiya N, Hotta N, Mitsufuji S, Nakamura M, Omori T, Maeda K, et al. Multicenter feasibility study of bowel preparation with castor oil for colon capsule endoscopy. Dig Endosc. 2019;31:164-72.

28. Conrad K, Roggenbuck D, Laass MW. Diagnosis and classification of ulcerative colitis. Autoimmun Rev. 2014;13:463-6.

29. Samuel S, Bruining DH, Loftus EV Jr, Becker B, Fletcher JG, Mandrekar JN, et al. Endoscopic skipping of the distal terminal ileum in Crohn's disease can lead to negative results from ileocolonoscopy. Clin Gastroenterol Hepatol. 2012;10:1253-9.

\section{Publisher's Note}

Springer Nature remains neutral with regard to jurisdictional claims in published maps and institutional affiliations.
Ready to submit your research? Choose BMC and benefit from:

- fast, convenient online submission

- thorough peer review by experienced researchers in your field

- rapid publication on acceptance

- support for research data, including large and complex data types

- gold Open Access which fosters wider collaboration and increased citations

- maximum visibility for your research: over $100 \mathrm{M}$ website views per year

At BMC, research is always in progress.

Learn more biomedcentral.com/submissions 